\title{
On the length of the continued fraction for values of quotients of power sums
}

\author{
par Pietro CORVAJA et UMBERTo ZANNIER
}

\begin{abstract}
RÉsumÉ. En généralisant un résultat de Pourchet, nous démontrons que si $\alpha, \beta$ sont deux sommes de puissances définies sur $\mathbb{Q}$, satisfaisant certaines conditions nécessaires, la longueur de la fraction continue pour $\alpha(n) / \beta(n)$ tend vers l'infini pour $n \rightarrow \infty$. On déduira ce résultat d'une inégalité de type Thue uniforme pour les approximations rationnelles des nombres de la forme $\alpha(n) / \beta(n)$.
\end{abstract}

Abstract. Generalizing a result of Pourchet, we show that, if $\alpha, \beta$ are power sums over $\mathbb{Q}$ satisfying suitable necessary assumptions, the length of the continued fraction for $\alpha(n) / \beta(n)$ tends to infinity as $n \rightarrow \infty$. This will be derived from a uniform Thue-type inequality for the rational approximations to the rational numbers $\alpha(n) / \beta(n), n \in \mathbb{N}$.

Introduction. The features of the continued fraction of a positive real number are usually extraordinarily difficult to predict. However, if the numbers in question run through certain parametrized families, some regularity occasionally appears. For instance, we may recall remarkable results by Schinzel [S1], [S2], on the lengths of the periods of the continued fractions for $\sqrt{f(n)}$, where $f$ is a polynomial with rational coefficients and $n$ varies through $\mathbb{N}$.

In a similar direction, but in a much more simpler way, one can deal with the continued fractions of the rational numbers $r(n), n \in \mathbb{N}$, where now $r \in \mathbb{Q}(X)$ is a rational function; for example, we may now look at the length of the fraction: in [S2, Lemma 2], Schinzel proves that this length is bounded as a function of $n$ (see also [M] for a more precise result). Substantially, the reason for this is that $r(X)$ has a finite expansion as a simple continued fraction with partial quotients in $\mathbb{Q}[X]{ }^{1}$

More generally, one may inquire about the length of the continued fraction for the rational values of other arithmetical functions. In general, due to the lack of an Euclid's algorithm, we have not a suitable "functional

\footnotetext{
Manuscrit reçu le 4 février 2004.

${ }^{1}$ Observe however that such continued fraction specialized at $X=n$ may not coincide with the continued fraction for $r(n)$.
} 
continued fraction"; this fact makes the situation different from the case of $\mathbb{Q}(X)$ and generally speaking considerably more difficult.

For certain exponential functions, we have an appealing question of Mendès-France (see [M2, p. 214]), stating that, for coprime integers $a, b>$ 1 , the length of the continued fraction for $(a / b)^{n}$ tends to infinity as $n \rightarrow \infty$, in marked contrast to the rational function case. This was answered affirmatively by Pourchet (unpublished) and independently by Choquet (in a weaker form); we refer to the paper [M3] for a discussion and several references. We also mention a paper by Grisel [G] solving a function-field analogue.

This theorem of Pourchet may be derived from the (deep) lower bound $\left|(a / b)^{n}-(p / q)\right| \gg_{\epsilon} q^{-2} \exp (-\epsilon n)$ (any positive $\epsilon$ ), where $p, q$ are integers with $0<q<b^{n}$ (which follows e.g. from Ridout generalization of Roth's Theorem $[R])$. The estimate is in fact amply sufficient, since it implies that all the partial quotients of the continued fraction in question are $\ll_{\epsilon}$ $\exp (\epsilon n)$, and the statement about the length follows at once. (See also [Z, Ex. II.6].)

In this direction, one may consider more general power sums in place of $a^{n}, b^{n}$. Namely, in analogy to [CZ1] (where the notation is slightly different) we consider the ring (actually a domain) $\mathscr{E}$ made up of the functions on $\mathbb{N}$ of the form

$$
\alpha(n)=c_{1} a_{1}^{n}+\ldots+c_{r} a_{r}^{n}
$$

where the coefficients $c_{i}$ are rational numbers, the $a_{i}$ are positive integers and the number $r$ of summands is unrestricted. (We shall often normalize (1) so that the $c_{i}$ are nonzero and the $a_{i}$ are distinct; in this case the $a_{i}$ are called the "roots" of $\alpha$.)

We then consider the ratio $\alpha / \beta$ of nonzero elements of $\mathscr{E}$ and ask about the length of the continued fraction for the values $\alpha(n) / \beta(n)$ (provided $\beta(n) \neq 0$, which is the case for all large $n$ ). It turns out (Corollary 2 below) that the length of the relevant continued fraction does not tend to infinity if and only if $\alpha / \beta$ admits an expansion as a (finite) continued fraction over $\mathscr{E}$. Note that $\mathscr{E}$ is not euclidean, so in practice this condition "rarely" holds.

This result plainly generalizes Pourchet's, but now the Ridout Theorem seems no longer sufficient for a proof. In fact, we shall need the full force of the Schmidt Subspace Theorem, similarly to [CZ1], where (among others) the related question of the integrality of the values $\alpha(n) / \beta(n)$ was investigated.

Similarly to the above sketched argument for Pourchet's Theorem, the conclusions about the continued fraction will be derived from a "Thue-type" inequality for the rational approximations to the values in question, which is the content of the Theorem below. Its proof follows [CZ1] in the main 
lines, except for a few technical points; however the present new simple applications of those principles are perhaps not entirely free of interest.

Preliminary to the statements, we introduce a little more notation.

We let $\mathscr{E} \mathbb{Q}$ be the domain of the functions of type (1), but allowing the $a_{i}$ to be positive rationals. If $\alpha \in \mathscr{E} \mathbb{Q}$ is written in the form (1), with nonzero $c_{i}$ and distinct $a_{i}$, we set $\ell(\alpha)=\max \left(a_{1}, \ldots, a_{r}\right)$, agreeing that $\ell(0)=0$.

It is immediate to check that

$$
\begin{aligned}
\ell(\alpha \beta) & =\ell(\alpha) \ell(\beta), \\
\ell(\alpha+\beta) & \leq \max (\ell(\alpha), \ell(\beta))
\end{aligned}
$$

and that $\ell(\alpha)^{n} \gg|\alpha(n)| \gg \ell(\alpha)^{n}$ for $n \in \mathbb{N}$ tending to infinity (so in particular, $\alpha(n)$ cannot vanish infinitely often if $\alpha \neq 0)$.

Theorem. Let $\alpha, \beta \in \mathscr{E}$ be nonzero and assume that for all $\zeta \in \mathscr{E}$, $\ell(\alpha-\zeta \beta) \geq \ell(\beta)$. Then there exist $k=k(\alpha, \beta)>0$ and $Q=Q(\alpha, \beta)>1$ with the following properties. Fix $\epsilon>0$; then, for all but finitely many $n \in \mathbb{N}$ and for integers $p, q, 0<q<Q^{n}$, we have

$$
\left|\frac{\alpha(n)}{\beta(n)}-\frac{p}{q}\right| \geq \frac{1}{q^{k}} \exp (-\epsilon n) .
$$

We tacitly mean that the alluded finite set of exceptional integers includes those $n \in \mathbb{N}$ with $\beta(n)=0$; this finite set may depend on $\epsilon$.

Remarks. (i) The assumption about $\alpha, \beta$ expresses the lack of an "Euclid division" in $\mathscr{E}$ for $\alpha: \beta$ and cannot be omitted. In fact, suppose that $\ell(\alpha-\zeta \beta)<\ell(\beta)$ for some $\zeta \in \mathscr{E}$. Then, the values $\zeta(n)$ have bounded denominator and verify $|(\alpha(n) / \beta(n))-\zeta(n)| \ll \exp \left(-\epsilon_{0} n\right)$ for large $n$ and some positive $\epsilon_{0}$ independent of $n$, against the conclusion of the Theorem (with $p / q=p_{n} / q_{n}=\zeta(n)$ ).

Note that the assumption is automatic if $\ell(\alpha) \geq \ell(\beta)$ and $\ell(\beta)$ does not divide $\ell(\alpha)$.

(ii) By the short argument after Lemma 1 below, given $\alpha, \beta$, one can test effectively whether a $\zeta \in \mathscr{E}$ such that $\ell(\alpha-\zeta \beta)<\ell(\beta)$ actually exists. Also, the proof will show that a suitable $Q$ and "exponent" $k$ may be computed. On the contrary, the finitely many exceptional $n$ 's cannot be computed with the present method of proof.

(iii) Naturally, the lower bound is not significant for $q$ larger than $\ell(\beta)^{n /(k-1)}$. So, some upper bound $Q^{n}$ for $q$ is not really restrictive. (Also, since the dependence of $k$ on $\alpha, \beta$ is unspecified, and since $\ell(\beta)^{1 /(k-1)} \rightarrow 1$ as $k \rightarrow \infty$, it is immaterial here to specify a suitable $Q$ in terms of $k$.) 
Note also that a lower bound $\gg Q^{n}$ for the denominator of $\alpha(n) / \beta(n)$ follows, sharpening [CZ1, Thm. 1]; in this direction, see [BCZ], [CZ3, Remark 1] and [Z, Thm. IV.3] for stronger conclusions in certain special cases.

(iv) Like for the proofs in [CZ1], the method yields analogous results for functions of the form (1), but where $a_{i}$ are any algebraic numbers subject to the sole (but crucial) restriction that there exists a unique maximum among the absolute values $\left|a_{i}\right|$. Using the (somewhat complicated) method of [CZ2], one may relax this condition, assuming only that not all the $\left|a_{i}\right|$ are equal. For the sake of simplicity, we do not give the proofs of these results, which do not involve new ideas compared to [CZ1], [CZ2] and the present paper, but only complication of detail.

(v) The Theorem can be seen as a Thue-type inequality with moving targets (similarly to [C] or [V]). It seems an interesting, but difficult, problem, to obtain (under suitable necessary assumptions) the "Roth's exponent" $k=2$, or even some exponent independent of $\alpha, \beta$. (As recalled above, this holds in the special cases of Pourchet's Theorem.)

Corollary 1. Let $\alpha, \beta$ be as in the Theorem. Then the length of the continued fraction for $\alpha(n) / \beta(n)$ tends to infinity as $n \rightarrow \infty$.

This corollary is in fact a lemma for the following result, which gives a more precise description.

Corollary 2. Let $\alpha, \beta \in \mathscr{E}$ be nonzero. Then the length of the continued fraction for $\alpha(n) / \beta(n)$ is bounded for infinitely many $n \in \mathbb{N}$ if and only if there exist power sums $\zeta_{0}, \ldots, \zeta_{k} \in \mathscr{E}$ such that we have the identical continued fraction expansion

$$
\frac{\alpha(n)}{\beta(n)}=\left[\zeta_{0}(n), \zeta_{1}(n), \ldots, \zeta_{k}(n)\right] .
$$

If this is the case, the mentioned length is uniformly bounded for all $n \in \mathbb{N}$.

It will be pointed out how the condition on $\alpha / \beta$ may be checked effectively.

The special case $\alpha(n)=a^{n}-1, \beta(n)=b^{n}-1$ appears as [Z, Ex. IV.12]. For completeness we give here this application.

Corollary 3. Let $a, b$ be multiplicatively independent positive integers. Then the length of the continued fraction for $\left(a^{n}-1\right) /\left(b^{n}-1\right)$ tends to infinity with $n$. 
Proofs. We start with the following very simple:

Lemma 1. Let $\alpha, \beta \in \mathscr{E}$ be nonzero and let $t$ be any positive number. Then there exists $\eta \in \mathscr{E}_{\mathbb{Q}}$ such that $\ell(\alpha-\eta \beta)<t$. Such an $\eta$ may be computed in terms of $\alpha, \beta, t$.

Proof. Write $\beta(n)=c b^{n}(1-\delta(n))$, where $c \in \mathbb{Q}^{*}, b=\ell(\beta)$ and where $\delta \in \mathscr{E}_{\mathbb{Q}}$ satisfies $u:=\ell(\delta)<1$. In particular, we have $|\delta(n)| \ll u^{n}$, so for a fixed integer $R$ we have an approximation, for $n \rightarrow \infty$,

$$
\frac{\alpha(n)}{\beta(n)}=\frac{\alpha(n)}{c b^{n}}\left(\sum_{r=0}^{R} \delta(n)^{r}\right)+O\left(\left(\frac{\ell(\alpha) u^{R+1}}{b}\right)^{n}\right) .
$$

Choose $R$ so that $u^{R}<t / u \ell(\alpha)$ and define $\eta$ by the first term on the right. Then $\eta \in \mathscr{E}_{\mathbb{Q}}$ and $|\alpha(n)-\eta(n) \beta(n)| \ll\left(\ell(\alpha) u^{R+1}\right)^{n}$. Therefore $\ell(\alpha-\eta \beta) \leq \ell(\alpha) u^{R+1}<t$, concluding the proof.

We note at once that this argument may be used to check the assumption for the Theorem. In fact, suppose $\ell(\alpha-\zeta \beta)<\ell(\beta)$ for a $\zeta \in \mathscr{E}$. Then, if $\eta$ is as in the lemma, with $t=\ell(\beta)$, it follows that $\ell(\eta-\zeta)<1$. Since $\eta$ may be constructed and since the "roots" of $\zeta$ are positive integers by assumption, this inequality determines $\zeta$ uniquely; namely, if a suitable $\zeta$ exists, it is just the "subsum" of $\eta$ made up with the integer roots.

To go on, for the reader's convenience, we recall a version of Schmidt's Subspace Theorem suitable for us; it is due to H.P. Schlickewei (see [Schm2, Thm.1E, p.178]).

Subspace Theorem. Let $S$ be a finite set of places of $\mathbf{Q}$, including the infinite one and normalized in the usual way (i.e. $|p|_{v}=p^{-1}$ if $v \mid p$ ). For $v \in S$ let $L_{0 v}, \ldots, L_{h v}$ be $h+1$ linearly independent linear forms in $h+1$ variables with rational coefficients and let $\delta>0$. Then the solutions $\mathbf{x}:=$ $\left(x_{0}, \ldots, x_{h}\right) \in \mathbf{Z}^{h+1}$ to the inequality

$$
\prod_{v \in S} \prod_{i=0}^{h}\left|L_{i v}(\mathbf{x})\right|_{v} \leq\|\mathbf{x}\|^{-\delta}
$$

where $\|\mathbf{x}\|:=\max \left\{\left|x_{i}\right|\right\}$, are all contained in finitely many proper subspaces of $\mathbf{Q}^{h+1}$.

For our last lemma we could invoke results by Evertse [E]; for completeness we give a short proof of the special case we need.

Lemma 2. Let $\zeta \in \mathscr{E}_{\mathbb{Q}}$ and let $D$ be the minimal positive integer such that $D^{n} \zeta(n) \in \mathscr{E}$. Then, for every $\epsilon>0$ there are only finitely many $n \in \mathbb{N}$ such that the denominator of $\zeta(n)$ is smaller than $D^{n} \exp (-\epsilon n)$. 
Proof. We may write $D^{n} \zeta(n)=\sum_{i=0}^{h} u_{i} e_{i}^{n}$, where $u_{i} \in \mathbb{Q}^{*}$ and $e_{i}$ are distinct positive integers with $\left(e_{0}, \ldots, e_{h}, D\right)=1$. Let $S$ be the set of places of $\mathbb{Q}$ made up of the infinite one and of those dividing $D e_{0} \cdots e_{h}$.

Define the linear form $L(\mathbf{X}):=\sum_{i=0}^{h} u_{i} X_{i}$.

For each $v \in S$ we define linear forms $L_{i v}, i=0, \ldots, h$, as follows. If $v$ does not divide $D$ (including $v=\infty$ ), then we put simply $L_{i v}=X_{i}$ for $i=0, \ldots, h$. If $v \mid D$, then there exists $j=j_{v}$ such that $\left|e_{j}\right|_{v}=1$; then we set $L_{j v}=L$ and $L_{i v}=X_{i}$ for $i \neq j$. The forms $L_{i v}, i=0, \ldots, h$, are plainly linearly independent for each fixed $v \in S$.

Suppose now that the conclusion does not hold, so for some positive $\epsilon$ and for all $n$ in an infinite set $\Sigma \subset \mathbb{N}$ the denominator of $\zeta(n)$ is $\leq D^{n} \exp (-\epsilon n)$. For $n \in \Sigma$, put $\mathbf{x}=\mathbf{x}(n)=\left(e_{0}^{n}, \ldots, e_{h}^{n}\right)$.

Then, for $n \in \Sigma$, the numerator of $D^{n} \zeta(n)$ has a g.c.d. with $D^{n}$ which is $\geq \exp (\epsilon n)$. In turn this gives

$$
\prod_{v \mid D}|L(\mathbf{x}(n))|_{v} \leq \exp (-\epsilon n), \quad n \in \Sigma .
$$

Also, for $n \in \Sigma$, we have

$$
\prod_{v \in S} \prod_{i=0}^{h}\left|L_{i v}(\mathbf{x})\right|_{v}=\left(\prod_{v \mid D}|L(\mathbf{x})|_{v}\right) \prod_{v \in S} \prod_{i=0}^{h}\left|e_{i}^{n}\right|_{v}=\prod_{v \mid D}|L(\mathbf{x})|_{v},
$$

where the first equality holds because $\left|e_{j_{v}}\right|_{v}=1$ for $v \mid D$ and the second because the (inner) double product is 1 by the product formula. Hence

$$
\prod_{v \in S} \prod_{i=0}^{h}\left|L_{i v}(\mathbf{x})\right|_{v} \leq \exp (-\epsilon n), \quad n \in \Sigma .
$$

Since however $\|\mathbf{x}(n)\|$ is bounded exponentially in $n$, we may apply the Subspace Theorem (with a suitable positive $\delta$ ) and conclude that all the $\mathbf{x}(n), n \in \Sigma$, lie in a finite union of proper subspaces. But $e_{0}, \ldots, e_{h}$ are distinct positive integers, whence each subspace corresponds to at most finitely many $n$, a contradiction which concludes the proof.

Proof of Theorem. Let $\eta$ be as in Lemma 1, with $t=1 / 9$. Write

$$
\eta(n)=g_{1}\left(\frac{e_{1}}{d}\right)^{n}+\ldots+g_{h}\left(\frac{e_{h}}{d}\right)^{n},
$$

where $g_{i} \in \mathbb{Q}^{*}$ and where $e_{i}, d$ are positive integers with $e_{1}>e_{2}>\ldots>e_{h}$.

We define $k:=h+3, Q=\exp (1 / k)$, and we assume that for some fixed $\epsilon>0$ (which we may take $<1 / 6$, say) there exist infinitely many triples $(n, p, q)$ of integers with $0<q \leq Q^{n}, n \rightarrow \infty$ and

$$
\left|\frac{\alpha(n)}{\beta(n)}-\frac{p}{q}\right| \leq \frac{1}{q^{k}} \exp (-\epsilon n) .
$$


We shall eventually obtain a contradiction, which will prove what we want.

We proceed to define the data for an application of the Subspace Theorem. We let $S$ be the finite set of places of $\mathbb{Q}$ consisting of the infinite one and of all the places dividing $d e_{1} \cdots e_{h}$.

We define linear forms in $X_{0}, \ldots, X_{h}$ as follows. For $v \neq \infty$ or for $i \neq 0$ we set simply $L_{i v}=X_{i}$. We define the remaining form $L_{0 \infty}$ by

$$
L_{0 \infty}(\mathbf{X})=X_{0}-g_{1} X_{1}-\ldots-g_{h} X_{h} .
$$

Plainly, for each $v$ the forms $L_{i v}$ are independent. For a triple $(n, p, q)$ as above we set

$$
\mathbf{x}=\mathbf{x}(n, p, q)=\left(p d^{n}, q e_{1}^{n}, \ldots, q e_{h}^{n}\right) \in \mathbb{Z}^{h+1}
$$

and we proceed to estimate the double product $\prod_{v \in S} \prod_{i=0}^{h}\left|L_{i v}(\mathbf{x})\right|_{v}$.

For $i \neq 0$ we have $\prod_{v \in S}\left|L_{i v}(\mathbf{x})\right|_{v}=\prod_{v \in S}\left|q e_{i}^{n}\right|_{v} \leq q$. In fact, we have $\prod_{v \in S}\left|e_{i}^{n}\right|_{v}=1$ by the product formula, since the $e_{i}$ are $S$-units; also, $\prod_{v \in S}|q|_{v} \leq|q|$, since $q$ is an integer.

Further, we have $\prod_{v \in S}\left|L_{0 v}(\mathbf{x})\right|_{v}=\frac{\left|L_{0 \infty}(\mathbf{x})\right|}{\left|x_{0}\right|} \prod_{v \in S}\left|x_{0}\right|_{v}$. As before, we see that the inner product is bounded by $|p|$, since $d^{n}$ is an $S$-unit.

Also, $\left|L_{0 \infty}(\mathbf{x})\right|=q d^{n}|\eta(n)-(p / q)|$. Then, using $(2)$ and $\mid(\alpha(n) / \beta(n))-$ $\eta(n) \mid \ll(t / \ell(\beta))^{n} \leq t^{n}$, we obtain

$$
\left|L_{0 \infty}(\mathbf{x})\right| \ll q d^{n} t^{n}+q d^{n}\left(q^{-k} \exp (-\epsilon n)\right) .
$$

Since $q^{k} \leq Q^{k n}=\exp (n)$ and since $t=1 / 9<\exp (-1-\epsilon)$, the first term on the right does not exceed the second one, whence

$$
\left|L_{0 \infty}(\mathbf{x})\right| \ll q d^{n}\left(q^{-k} \exp (-\epsilon n)\right) .
$$

Combining this with the previous bound we get

$$
\prod_{v \in S}\left|L_{0 v}(\mathbf{x})\right|_{v} \ll q d^{n}\left(q^{-k} \exp (-\epsilon n)\right)\left|x_{0}\right|^{-1}|p|=q^{-(k-1)} \exp (-\epsilon n) .
$$

Finally, for the double product this yields (since $k>h+1$ )

$$
\prod_{v \in S} \prod_{i=0}^{h}\left|L_{i v}(\mathbf{x})\right|_{v} \ll q^{-(k-h-1)} \exp (-\epsilon n) \ll \exp (-\epsilon n) .
$$

Now, (2) entails $|p| \ll q \ell(\alpha)^{n} \ll(Q \ell(\alpha))^{n}$, whence $\|\mathbf{x}\| \ll C^{n}$ for some $C$ independent of $n$. Hence the double product is bounded by $\|\mathbf{x}\|^{-\delta}$ for some fixed positive $\delta$ and large enough $n$.

The Subspace Theorem then implies that all the vectors $\mathbf{x}$ in question are contained in a certain finite union of proper subspaces of $\mathbb{Q}^{h+1}$.

In particular, there exists a fixed subspace, say of equation $z_{0} X_{0}-z_{1} X_{1}-$ $\ldots-z_{h} X_{h}=0$, containing an infinity of the vectors in question. We cannot have $z_{0}=0$, since this would entail $\sum_{i=1}^{h} z_{i} e_{i}^{n}=0$ for an infinity of $n$; in 
turn, the fact that the $e_{i}$ are distinct would imply $z_{i}=0$ for all $i$, a contradiction. Therefore we may assume that $z_{0}=1$, and we find that, for the $n$ 's corresponding to the vectors in question,

$$
\frac{p}{q}=\sum_{i=1}^{h} z_{i}\left(\frac{e_{i}}{d}\right)^{n}=\zeta(n),
$$

say, where $\zeta \in \mathscr{E} \mathbb{Q}$. We now show that actually $\zeta$ lies in $\mathscr{E}$. Assume the contrary; then the minimal positive integer $D$ so that $D^{n} \zeta(n) \in \mathscr{E}$ is $\geq 2$. But then equation (3) together with Lemma 2 implies that $q \gg$ $2^{n} \exp (-\epsilon n)$. Since this would hold for infinitely many $n$, we would find $Q \geq 2 \exp (-\epsilon)$, whence $\exp ((1 / k)+\epsilon) \geq 2$, a contradiction. (Recall that $k \geq 3, \epsilon<1 / 6$.)

Therefore $\zeta$ lies in $\mathscr{E}$; using (3) to substitute in (2) for $p / q$ we find that, for an infinity of $n$,

$$
\left|\frac{\alpha(n)}{\beta(n)}-\zeta(n)\right| \leq \exp (-\epsilon n) .
$$

In particular, $\ell((\alpha / \beta)-\zeta)<1$, whence $\ell(\alpha-\zeta \beta)<\ell(\beta)$, contrary to the assumptions, concluding the proof.

Proof of Corollary 1. For notation and basic facts about continued fractions we refer to [Schm1, Ch. I] and let $p_{r}(n) / q_{r}(n), r=0,1, \ldots$, be the (finite) sequence of convergents of the continued fraction for $\alpha(n) / \beta(n)$, where we may assume that $\alpha(n)$ and $\beta(n)$ are positive.

As is well-known, $p_{r}(n), q_{r}(n)$ are positive integers, $q_{0}(n)=1$, and we have

$$
\left|\frac{\alpha(n)}{\beta(n)}-\frac{p_{r}(n)}{q_{r}(n)}\right| \leq \frac{1}{q_{r+1}(n) q_{r}(n)} .
$$

Suppose the conclusion false, so for some fixed $R \in \mathbb{N}$ there is an infinite set $\Sigma \subset \mathbb{N}$ such that for $n \in \Sigma$ the continued fraction for $\alpha(n) / \beta(n)$ has finite length $R+1 \geq 1$. By this we mean that $p_{R}(n) / q_{R}(n)=\alpha(n) / \beta(n)$.

Since $\alpha, \beta$ satisfy the assumptions for the Theorem, there exist $Q>1$, $k \geq 2$ as in that statement.

Define now the sequence $c_{0}, c_{1}, \ldots$ by $c_{0}=0$ and $c_{r+1}=(k-1) c_{r}+1$ and choose a positive number $\epsilon<c_{R}^{-1} \log Q$, so $\exp \left(c_{R} \epsilon\right)<Q$; we proceed to show by induction on $r=0, \ldots, R$, that, for large $n \in \Sigma$,

$$
q_{r}(n) \leq \exp \left(c_{r} \epsilon n\right) .
$$

We have $q_{0}(n)=1$, so (5) is true for $r=0$; we shall now show that (if $r \leq R-1$ ), for large $n,(5)$ implies the same inequality with $r+1$ in place of $r$.

Observe first that, by construction, $\exp \left(c_{r} \epsilon\right) \leq \exp \left(c_{R} \epsilon\right)<Q$, whence $q_{r}(n) \leq \exp \left(c_{r} \in n\right)<Q^{n}$ for large enough $n \in \Sigma$. We may then apply the 
Theorem with $p=p_{r}(n), q=q_{r}(n)$ and deduce that for large $n \in \Sigma$ we have

$$
\left|\frac{\alpha(n)}{\beta(n)}-\frac{p_{r}(n)}{q_{r}(n)}\right| \geq q_{r}(n)^{-k} \exp (-\epsilon n) .
$$

Hence, combining with (4),

$$
q_{r+1}(n) \leq q_{r}(n)^{k-1} \exp (\epsilon n) \leq \exp \left(\left((k-1) c_{r}+1\right) \epsilon n\right)=\exp \left(c_{r+1} \epsilon n\right)
$$

by the inductive assumption and the definition of $c_{r+1}$. This induction proves (5) for $r \leq R$.

Finally, by (5) with $r=R$, we have $q_{R}(n) \leq \exp \left(c_{R} \epsilon n\right)$ for large $n \in \Sigma$, whence $q_{R}(n)<Q^{n}$, since $\exp \left(c_{R} \epsilon\right)<Q$ by construction. But then the Theorem holds for $p=p_{R}(n), q=q_{R}(n)$, leading in particular to $0=$ $\left|(\alpha(n) / \beta(n))-\left(p_{R}(n) / q_{R}(n)\right)\right|>0$, a contradiction which concludes the argument.

Proof of Corollary 2. For convenience let us denote by $\psi(x)$ the length of the continued fraction for the rational number $x$.

We start with the hardest half of the proof; namely, assuming that $\psi(\alpha(n) / \beta(n))$ is bounded for $n$ in an infinite sequence $\Sigma \subset \mathbb{N}$, we prove that $\alpha / \beta$ has a finite continued fraction over $\mathscr{E}$.

Let us argue by induction on $\ell(\alpha)+\ell(\beta)$, the result being trivial when this number is $\leq 2$.

Since $|\psi(x)-\psi(1 / x)| \leq 1$, we may plainly assume $\ell(\alpha) \geq \ell(\beta)$. By our present assumption, the hypothesis of Corollary 1 cannot hold for $\alpha, \beta$, so there exists $\zeta \in \mathscr{E}$ such that, putting $\eta=\alpha-\zeta \beta$, we have $\ell(\eta)<\ell(\beta)$. For large $n$, we have $\beta(n) \neq 0$ and

$$
\frac{\eta(n)}{\beta(n)}=\frac{\alpha(n)}{\beta(n)}-\zeta(n) .
$$

Since $\zeta \in \mathscr{E}$, the rational numbers $\zeta(n), n \in \mathbb{N}$, have a finite common denominator $D>0$. We now appeal to [S1, Thm. 1], which bounds $\psi((p / q)-r)$ in terms of $\psi(p / q)$, where $r$ is a rational number with fixed denominator. Applying this with $p / q=\alpha(n) / \beta(n), r=\zeta(n)$ and noting that $\psi(\alpha(n) / \beta(n))$ is bounded by assumption for $n \in \Sigma$, we immediately obtain that the numbers $\psi(\eta(n) / \beta(n))$ are also bounded for $n \in \Sigma$. Since $\ell(\eta)+\ell(\beta)<\ell(\alpha)+\ell(\beta)$, the inductive assumption implies the existence of a finite continued fraction expansion over $\mathscr{E}$ for $\eta / \beta$. In turn, this yields the same for $\alpha / \beta$, proving the conclusion. 1].

The "converse" part of Corollary 2 is again immediate from [S1, Thm.

Like for Corollary 1 , the condition that $\alpha / \beta$ admits a finite continued fraction over $\mathscr{E}$ may be checked effectively. To verify this, observe first that, if $\alpha / \beta$ admits a continued fraction over $\mathscr{E}$ as in the statement, then it also 
admits an expansion such that $\ell\left(\zeta_{i}\right) \geq 1$ for all $i \geq 1$; this claim follows at once from the continued fraction identity

$$
[A, c, B]=\left[A+c^{-1},-c(c B+1)\right],
$$

which allows to absorb all possible "constant" partial quotients $c$.

With this proviso (but not otherwise) the continued fraction over $\mathscr{E}$ is unique (if it exists); its existence may be checked e.g. by iterating the effective criterion for checking the assumption of the Theorem (or of Corollary $1)$, as in the comments after Lemma 1.

Proof of Corollary 3. We could invoke Corollary 2, but it is perhaps simpler to argue directly, by induction on $a+b$ (the assertion being empty for $a+b=2$ ). On exchanging $a, b$ we may assume that $a>b>1$.

Now, we may assume that $a$ is divisible by $b$; in fact, if this does not hold, we plainly have $\ell\left(a^{n}-1-\zeta\left(b^{n}-1\right)\right) \geq a>b$ for any $\zeta \in \mathscr{E}$; in turn, we may then apply ( $a$ fortiori) Corollary 1 to $\alpha(n)=a^{n}-1, \beta(n)=b^{n}-1$, yielding the present assertion.

Write then $a=b c$, where $c$ is a positive integer $<a$. Note that $b, c$ are multiplicatively independent (since $a, b$ are such) whence the continued fraction for $\left(c^{n}-1\right) /\left(b^{n}-1\right)$ has length tending to infinity, by the inductive assumption. But then the identity

$$
\frac{a^{n}-1}{b^{n}-1}=c^{n}+\frac{c^{n}-1}{b^{n}-1}
$$

immediately implies the sought conclusion.

Final remarks. (a) It seems a very difficult problem to quantify the corollaries, namely to prove an explicit lower bound for the length of the relevant continued fractions. Even in the special case of Pourchet's Theorem, this seems to be related with an explicit version of Ridout's (or Roth's) Theorem, which presently appears inaccessible.

(b) The methods of proof probably lead to results similar to the Theorem, where $\alpha(n) / \beta(n)$ is replaced by some algebraic function of a power sum, like e.g. the $d$-th root $\alpha(n)^{1 / d}$. The pattern should combine the arguments from, say, [CZ1, Cor. 1] (or [CZ4, Thm. 2]) with the above ones.

In case $d=2$ it is perhaps possible to obtain corollaries similar to the present one, but in the direction of Schinzel's mentioned papers; namely, under suitable assumptions on the power-sum $\alpha \in \mathscr{E}_{\mathbb{Q}}$, the length of the period of the continued fraction for $\sqrt{\alpha(n)}$ tends to infinity with $n$.

After the present paper was referred this problem was approached by A. Scremin, in the course of his $\mathrm{PhD}$ thesis. A paper will soon appear in Acta Arithmetica. In the meantime F. Luca and Y. Bugeaud have also worked 
on this problem; see their paper "On the period of the continued fraction expansion of $\sqrt{2^{2 n+1}+1}$ ". Indag. Math. (N.S.) 16 (2005), no. 1, 21-35.

Also, the present authors wish to thank prof. Y. Bilu and an anonymous referee for several references. This also gave to the present authors the occasion of learning some new questions in the context; for instance we found that the present methods may be relevant, leading e.g. to an answer to Problem 6 of [M3]; see our article "On the rational approximations to the powers of an algebraic number: Solution of two problems of Mahler and Mendès France". Acta Mathematica, 193.

\section{References}

[BCZ] Y. Bugeaud. P. Corvaja. U. Zannier, An upper bound for the G.C.D. of $a^{n}-1$ and $b^{n}-1$. Math. Z. 243 (2003), 79-84.

[C] P. CoRvajA, Une application nouvelle de la méthode de Thue. Annales de l'Institut Fourier 45 (1995), 1177-1203.

[CZ1] P. CoRvaja, U. ZANnIER, Diophantine equations with power sums and Universal Hilbert Sets. Indag. Mathem., N.S. 9 (3) (1998), 317-332.

[CZ2] P. CoRvaja, U. ZANniER, Finiteness of integral values for the ratio of two linear recurrences. Invent. Math. 149 (2002), 431-451.

[CZ3] P. Convaja, U. Zannier, On the greatest prime factor of $(a b+1)(a c+1)$. Proc. Amer. Math. Soc. 131 (2003), 1705-1709.

[CZ4] P. CorvajA. U. ZAnniER, Some New Applications of the Subspace Theorem. Compositio Math. 131 (2002), 319-340.

[E] Y.-H. EverTSE, On sums of $S$-units and linear recurrences. Compositio Math. 53 (1984), 225-244.

[G] G. GRISEL, Length of the powers of a rational fraction. J. Number Theory, 62 (1997), 322-337.

[M] M. MENDÈs-FrANCE, Quelques problèmes relatifs à la théorie des fractions continues limitées. Sém. Delange-Pisot-Poitou, 13e année (1971/72), Théorie des nombres, 1, Exp. No. 2, Secrétariat Mathématique, Paris, 1973.

[M2] M. MEndìs-France, Sur les fractions continues limitées. Acta Arith. 23 (1973), 207215.

[M3] M. MENDÈS-FRANCE, Remarks and problems on finite and periodic continued fractions. L'Enseignement Math. 39 (1993) 249-257.

[R] D. RIDOUT, The p-adic generalization of the Thue-Siegel-Roth theorem. Mathematika 5 (1958), 40-48.

[S1] A. SCHINZEL, On some problems of the arithmetical theory of continued fractions. Acta Arith. 6 (1961), 393-413.

[S2] A. SCHINZEL, On some problems of the arithmetical theory of continued fractions II. Acta Arith. 7 (1962), 287-298.

[Schm1] W.M. SchmidT, Diophantine Approximation. Springer LNM 785, 1980.

[Schm2] W.M. SснміDT, Diophantine Approximations and Diophantine Equations. Springer Verlag LN 1467, 1991.

[V] P. VoJTA, Roth's theorem with moving targets. Internat. Math. Res. Notices 3 (1996) 109-114.

[Z] U. ZANnIER, Some applications of Diophantine Approximation to Diophantine Equations. Forum Editrice, Udine, 2003. 
Pietro CoRvaja

Dipartimento di Matematica

Università di Udine

via delle Scienze 206

33100 Udine (Italy)

E-mail : corvaja@dimi.uniud.it

Umberto ZANNIER

Scuola Normale Superiore

Piazza dei Cavalieri, 7

56100 Pisa (Italy)

E-mail : u.zannier@sns.it 